Copyright $(2008$ Institute of Electrical and Electronics Engineers, Inc.

All rights reserved.

Personal use of this material, including one hard copy reproduction, is permitted.

Permission to reprint, republish and/or distribute this material in whole or in part for any other purposes must be obtained from the IEEE.

For information on obtaining permission, send an e-mail message to stds-ipr@ieee.org.

By choosing to view this document, you agree to all provisions of the copyright laws protecting it.

Individual documents posted on this site may carry slightly different copyright restrictions.

For specific document information, check the copyright notice at the beginning of each document. 


\title{
Clustering and Least Square based Neural Technique for Learning and Identification of Suspicious Areas within Digital Mammograms
}

\author{
Peter McLeod and Brijesh Verma \\ School of Computing Sciences, Central Queensland University \\ North Rockhampton, QLD 4701, Australia \\ peterm@practical.com.au,b.verma@cqu.edu.au
}

\begin{abstract}
This paper presents a technique which explores the fusion of clustering and a least square method for the classification of suspicious areas within digital mammograms into benign and malignant classes. It incorporates a clustering algorithm such as $k$-means in conjunction with a gram-schmidt based least square method. The main focus of the research presented in this paper is to (1) improve the classification of features from suspicious areas within digital mammograms and (2) examine the effects that the determined clusters and least square methods have on classification accuracy and efficiency. The proposed technique has been tested on a benchmark database and the results from preliminary experiments are discussed.
\end{abstract}

\section{Introduction}

Breast Cancer is a leading cause of mortality and morbidity [1-2] amongst women. A report by a National Cancer Institute [2] suggests that 1 in 11 women develop breast cancer in Australia, 1 in 8 in the United States and 1 in 9 in the United Kingdom and Canada. Since the aetiology of breast cancer is not known prevention at this stage seems unlikely which makes early detection more important.

Digital mammography is one of the most reliable procedures for the early detection of breast cancer [1-2, 32-33]. Current image processing techniques [33] make primitive breast abnormality detection easier; however their classification as malignant or benign remains one of the most difficult tasks for radiologists and researchers due to a lack of individuality in benign and malignant class patterns. In many cases, both classes exhibit similar characteristics, i.e. size, shape and distribution of microcalcifications. Radiologists' interpretation of such cases often produce screening errors; either to miss malignant cases or result in more benign biopsies. The ability of neural classifiers to learn from the attributes of given class patterns and to classify unknown patterns into appropriate classes using the acquired knowledge has shown its potential $[3-10,20,23,25,27,29,31]$ in the field of digital mammography.

Techniques such as artificial neural networks [4-9], fuzzy logic [4, 11], and wavelet transforms [12-13] are the most commonly used detection and classification systems. Chitre et al. [9] compared artificial neural networks and statistical methods for microcalcification pattern classification. They obtained a classification rate of $60 \%$, which was better than statistical classifiers. A comparative study of a radial basis function (RBF) and a multi layer perceptron (MLP) neural networks for the classification of breast abnormalities using texture features was performed by Christoyianni et al. [10] and Bovis et al. [8]. They concluded that MLP obtained $4 \%$ higher accuracy than RBF. Yu et al. [16] used a multilayer feed forward neural network. 
Verma et al. [4] used a back-propagation neural network for the classification of suspicious lesions extracted using a fuzzy rule based detection system. They obtained an $88.9 \%$ classification rate using a manual combination of features. Zhang et al. [5] used a genetic algorithm for neural network learning in their study of microcalcification classification in digital mammograms. They have attained a $90.5 \%$ accuracy rate on test set at the cost of a low accuracy rate on training set. Wroblewska et al. [14] proposed a new segmentation and feature extraction technique for the reliable classification of microcalcifications which achieved low classification rate (78\%) on DDSM database.

A critical review of existing techniques [3-33] has shown that the accuracy rate for the classification of breast abnormalities is low and inconsistent. There are many reasons for low classification rates such as inappropriate training of classifiers, contradictory features which confuse classifiers, inconsistent feature values for benign and malignant suspicious areas, etc. The previous research showed that neural classifiers can achieve better performance [3-5, 8-10, 20] than other traditional techniques in classifying benign and malignant patterns in digital mammography. Recent reviews [3, 20] have reported the superiority of neural networks over other techniques. However, there are many drawbacks with current neural classifiers for the diagnosis of breast cancer.

The primary aim of this study is to incorporate an unsupervised clustering algorithm (kmeans clustering) with a least square method for determining clusters and weights for neural network based classifiers. These weights are used by a supervised learning algorithm to classify the features extracted from suspicious areas into malignant and benign classes.

The remainder of this paper is organised into four sections. Section 2 discusses the proposed research methodology. Section 3 presents the experimental results obtained with the proposed approach. Section 4 covers a brief discussion and analysis on the experimental results obtained with the proposed methodology. In section 5, conclusions are drawn and future research directions are addressed.

\section{Proposed Research Methodology}

An overview of the research methodology is presented below in Figure 1. The research methodology consists of data collection, suspicious area extraction, feature extraction/selection, clustering, learning and classification processes.

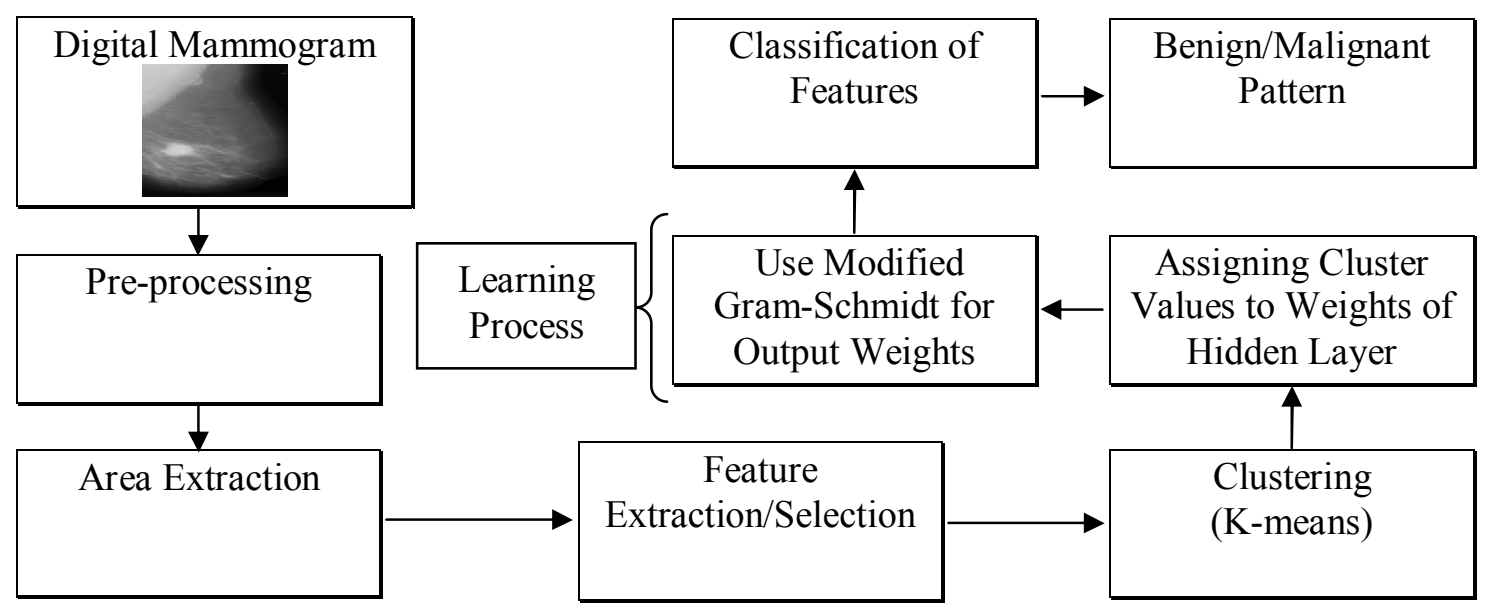

Figure 1. An overview of the proposed research methodology 
The first step is data/image acquisition. A generally accepted practice with the use of adaptive pattern recognition techniques is to use two sets of data in order to train, test and determine the suitability of the diagnostic system. The digital mammograms from the University of South Florida's Digital Database for Screening Mammography (DDSM) are used in this study to allow for comparisons with other researchers [17].

The pre-processing, area extraction and feature extraction techniques from our previous research work was used to extract appropriate features from digital mammograms. The k-means clustering algorithm was used to cluster input features into a number of clusters. The learned cluster values were used to assign the weights of hidden layer (weights between inputs and hidden units) of a MLP type neural network. The network contained inputs (features), hidden units (single hidden layer) and outputs (benign and malignant classes). The weights of the output layer (weights between hidden units and outputs) were calculated by a modified-gram Schmidt (MGS) based least square method. The weights obtained by clustering and MGS were used in conjunction with supervised network training to classify input features into benign and malignant classes. The proposed methodology provides the ability to quickly, efficiently, and accurately identify suspicious areas into benign and malignant classes.

\section{Experimental Results}

The proposed methodology has been implemented in $\mathrm{C}++$. The experimental results obtained from various experiments are presented below in Table 1. In our preliminary experiments the number of features and the number of clusters were fixed and the number of hidden units was varied. The 5-page limit does not allow us to include all results in Table 1 so we have included results for a selected number of hidden units. The best results were obtained with 28 hidden units.

Table 1. Classification Accuracy

\begin{tabular}{|c|c|c|c|c||}
\hline \multirow{2}{*}{$\begin{array}{c}\text { \# of } \\
\text { Features }\end{array}$} & \multirow{2}{*}{$\begin{array}{c}\text { \#of } \\
\text { Clusters }\end{array}$} & \multirow{2}{*}{$\begin{array}{c}\text { \#of } \\
\text { Hidden } \\
\text { Units }\end{array}$} & \multicolumn{2}{|c|}{$\begin{array}{c}\text { Classification Accuracy } \\
{[\%]}\end{array}$} \\
\cline { 4 - 5 } & & 19 & 95 & Training Set \\
\hline 6 & 8 & Testing Set \\
\hline 6 & 8 & 20 & 95 & 86 \\
\hline 6 & 8 & 24 & 95 & 87 \\
\hline 6 & 8 & 26 & 95 & 88 \\
\hline 6 & 8 & 28 & 95 & 91 \\
\hline
\end{tabular}

\section{Comparative Analysis}

The results obtained from the proposed methodology were analysed and compared with other existing techniques. The highest classification accuracy of $91 \%$ on test set was obtained using k-means and modified gram-schmidt for obtaining hidden and output weights. A comparison with other existing pattern classification systems is a difficult task as many systems are developed and tested on different datasets. Figure 2 shows the classification rates attained by our proposed research methodology and other researchers' proposed techniques. Bovis et al. [8] attained $77 \%$ classification accuracy with BPNN and 78\% with RBFNN on 161 breast images of the MIAS dataset. $\mathrm{Wu}$ et al. [7] used a dataset containing 500 masses from the China Society for Industrial and Applied Mathematics. They reported highest $87.77 \%$ classification accuracy using the weighted average fusion algorithm on balanced input patterns to their NN ensembles and $88.27 \%$ accuracy with the perceptron average fusion algorithm on imbalanced input 
patterns. Panchal et al. [33] used an autoassociater-MLP based classifier and they reported 91\% accuracy on a test set of the DDSM benchmark database, however the training of autoassociator and MLP took much longer than the proposed methodology.

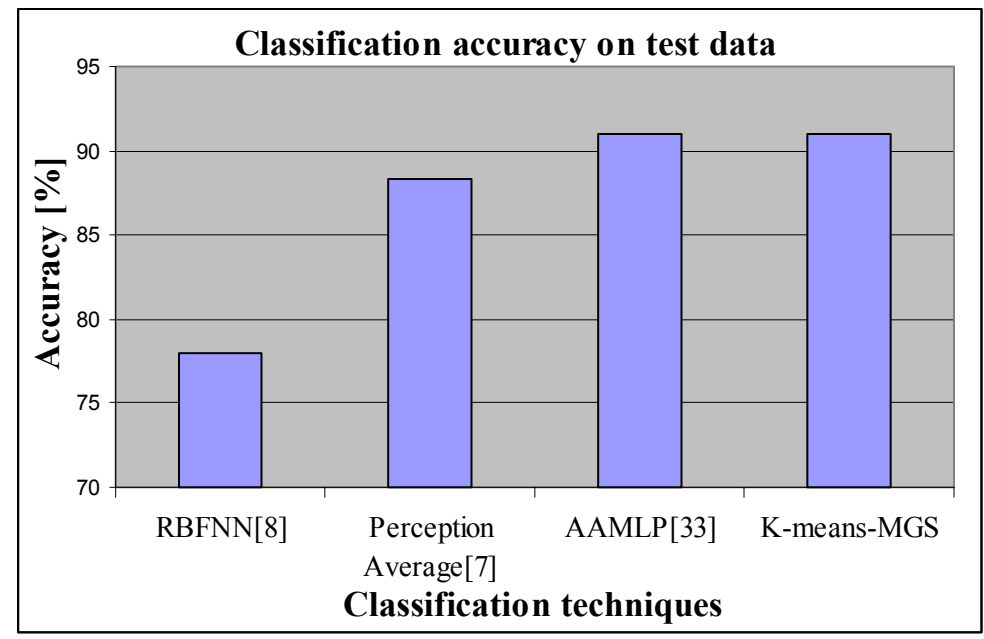

Figure 2. Proposed methodology vs. existing techniques

\section{Conclusion}

The research investigations presented in this paper has demonstrated that the combination of clustering algorithms with modified gram-schmidt can achieve good classification accuracy for benign and malignant patterns. The learning of hidden and output weights was very fast. The experiments showed that a small number of iterations was required for k-means and modified gram-schmidt to train the hidden and output weights of the network. Overall, the proposed approach is free from traditional problems faced by iterative learning processes and provides fast training with promising classification accuracy. The experiments were conducted with only one set of features and a fixed number of clusters. In our future research, we will conduct more experiments with various numbers of clusters and features. We are also planning to replace kmeans with other clustering algorithms such as self organising map and investigate its impact on the overall learning process and classification accuracy.

\section{References}

[1] Parkin, M., Freddie Bray, F, Ferlay, J. and Pisani, P. "Global Cancer Statistics", A Cancer Journal for Clinicians, Vol. 55, pp. 74-108, 2005, American Cancer Society

[2] National Breast Cancer Centre, http://www.nbcc.org.au/bestpractice/statistics/

[3] Cheng, H. D., Cai, X., Chen, X., Hu, L., and Lou, X., "Computer-aided Detection and Classification of Microcalcification in Mammograms: a survey”, Pattern Recognition,Vol. 36, pp. 2967-2991, 2003.

[4] Verma, B. and Zakos, J., "A Computer-Aided Diagnosis System for Digital Mammograms Based on FuzzyNeural and Feature Extraction Techniques”, IEEE Trans. on Info. Tech. in Biomedicine, Vol. 5, pp.46-54, 2001.

[5] Zhang P., Verma, B., and Kumar K., "A Neural-Genetic Algorithm for Feature Selection and Breast Abnormality Classification in Digital Mammography”, Proc. of IEEE-IJCNN, Vol. 3, pp. 2303-2309, 2004.

[6] Zhang, P., Verma, B. and Kumar, K. Neural Vs. Statistical Classifier in Conjunction with Genetic Algorithm Feature Selection in Digital Mammography, Pattern Recognition Letters, 26(7), pp. 909-919, 2005.

[7] Wu Y., He J., Man Y., and Arribas J. I., "Neural Network Fusion Strategies for Identifying Breast Masses", Proc. of IEEE-IJCNN, Vol. 3, pp. 2437-2442, 2004.

[8] Bovis K., Singh S., Fieldsend J., and Pinder C., "Identification of Masses in Digital Mammograms with MLP and RBF Nets", Proc. of IEEE-IJCNN, Vol. 1, pp. $342-347,2000$. 
[9] Chitre Y., Dhawan A. P., and Moskowitz M., "Artificial Neural Network Based Classification of Mammographic Microcalcifications using Image Structure Features", State of The Art in Digital Mammographic Image Analysis, World Scientific Publication, Vol. 9, pp. 167-197, 1994.

[10] Christoyianni L., Dermatas E., and Kokkinakis G., "Neural Classification of Abnormal Tissues in Digital Mammography using Statistical Features of the Texture”, Proc. Of IEEE-ICECS, Vol. 1, pp. 117-120, 1999.

[11] Cheng, H., Lui, Y. M., and Freimanis, R. I., "A Novel Approach to Microcalcification Detection using Fuzzy Logic Technique”, IEEE Trans. on Medical Imaging, Vol. 17, pp. 442-450, 1998.

[12] Yoshida H., Nishikawa R. N., Geiger M.L., and Doi. K., "Signal/Background Separation By Wavelet Packets for Detection of Microcalcifications in Mammograms", Proc. of SPIE on Wavelet Applications in Signal and Image Processing IV, Vol. 2825, pp 805-811, 1996.

[13] Wang T. C., and Karayiannis N. B., "Detection of Microcalcification in Digital Mammograms using Wavelets", IEEE Transactions on Medical Imaging, Vol. 17, pp. 498-509, 1998.

[14] Wroblewska A., Boninski P., Przelaskowski A., and Kazubek M., "Segmentation and feature extraction for reliable classification of microcalcification in digital mammograms", Opto-Electronics Review, Vol. 11, pp. 227-235, 2003.

[15] Markey M. K., Lo J. Y., and Floyd C. E., "Differences between Computer-aided Diagnosis of Breast Masses and That of Calcifications", Radiology, Vol 223, pp. 489-493, 2002.

[16] Yu S., and Guan L., "A CAD System for the Automatic Detection of Clustered Microcalcifications in Digitized Mammogram Films”, IEEE Trans. on Medical Imaging, Vol. 19, pp. 115-126, 2000.

[17] Heath M., Bowyer K., Kopans D., Moore R., and Kegelmeyer Jr. P., "The Digital Database for Screening Mammography”, IWDM-2000, Medical Physics Publishing, 2001.

[18] Rangayyan R. M., Mudigonda N. R., and Leo Desautels J. E., "Boundary modeling and shape analysis methods for classification of mammographic masses", Medical and Biological Eng. and Computing, Vol. 38, pp. 487496, 2000

[19] Verma, B. "Fast Training of Multilayer Perceptrons (MLPs)", IEEE Transactions on Neural Networks, vol. 8, no. 6, pp. 1314-1321, 1997.

[20] Verma, B. and Panchal, R. "Neural Networks for the Classification of Benign and Malignant Patterns in Digital Mammograms", Advances in Applied Artificial Intelligence, Idea Group, Inc., USA, Book Editor: John Fulcher, 2006.

[21] Kallergi M, Carney GM and Gaviria J. "Evaluating the Performance of Detection Algorithms in Digital Mammography”, Med Phys. 26(2):267-75, 1999. Pickard, J. "Using Receiver Operating Characteristic (ROC) Curves to Evaluate Digital " http://ric.uthscsa.edu/personalpages/lancaste/DI2_Projects_2004/JP_Project.pdf

[22] Markey, M., Lo, J., Tourassi, G., and Floyd, C., "Self-organizing Map for Cluster Analysis of a Breast Cancer Database", Artificial Intelligence in Medicine, 27, pp. 113-127, 2003.

[23] Lee, G., and Bottema, M., "Classification of Masses in Screening Mammograms as Benign or Malignant", IWDM2000, Canada, 2000, http://www.sunnybrook.on.ca/ iwdm2000/iwdm2000_abstract_book.pdf, pp. 52.

[24] Wu, Y., “Application of Neural Networks in Mammography”, Radiology, vol. 187, pp. 81-87, 1993.

[25] Bakic, P., and Barzakovic, D., "Application of Neural Networks in Computer Aided Diagnosis of Breast Cancer", Proceedings of 5th workshop on Neural Network Applications, NEUREL, Belgrade, Yugoslavia, 1997.

[26] Guran, M., Chan, H., Sahiner, B., Hadjiiski, L., Petrick, N., and Helvie, M., "Optimal Neural Network Architecture Selection: Improvement in Computerized Detection of Microcalcifications", Acad. Radiology. 9 (4), pp. 420-29, 2002.

[27] Guran, M., Sahiner, B., Chan, H., Hadjiiski, L., and Petrick, N., "Selection of an Optimal Neural Network Architecture for Computer-Aided Detection of Microcalcifications-Comparison of Automated Optimization Techniques", Med. Phys. 28 (9), pp. 1937-1948, 2001.

[28] Papadopoulos A., Fotiadis D. I., and Likas A., "An Automatic Microcalcification Detection System Based on a Hybrid Neural Network Classifier”, Artificial Intelligence in Medicine, Vol. 25, pp. 149-167, 2002.

[29] Lee, Y., and Tsai, D., "Computerized Classification of Microcalcifications on Mammograms using Fuzzy Logic and Genetic Algorithm”, Medical Imaging, Proc. of SPIE, Vol. 5370, pp 952-959, 2004.

[30] Khuwaja, G. A., and Abu-Rezq, A. N., "Bi-Model Breast Cancer Classification System, Pattern Analysis Applications", Springer-Verlag, Vol. 7, pp. 235-242, 2004.

[31] Lo, J. Y., Land W. H., Morrison, C. T, "Evolutionary Programming Technique for Reducing Complexity of Artificial Neural Networks for Breast Cancer Diagnosis", Proc. of SPIE 3979: 153-158, 2000.

[32] AbuBaker, A., Qahwaji, R., Aqel, M., Al-Osta, H. and Saleh, M., "Efficient Preprocessing of USF and MIAS Mammogram Images”, Journal of Computer Science, Vol. 3, No. 2, pp. 67-75, 2006.

[33] Panchal, R. and Verma, B., Neural Classification of Mass Abnormalities with Different types of Features in Digital Mammography, International Journal of Computational Intelligence and Applications, Vol. 6, No. 1, pp. 61-76, 2006. 